\title{
The structural biology of ryanodine receptors
}

\author{
Lynn KIMLICKA \& Filip VAN PETEGEM* \\ University of British Columbia, Department of Biochemistry and Molecular Biology, Vancouver V6T 1Z3, Canada
}

Received May 1, 2011; accepted May 30, 2011

\begin{abstract}
Ryanodine receptors are ion channels that allow for the release of $\mathrm{Ca}^{2+}$ from the endoplasmic or sarcoplasmic reticulum. They are expressed in many different cell types but are best known for their predominance in skeletal and cardiac myocytes, where they are directly involved in excitation-contraction coupling. With molecular weights exceeding 2 MDa, Ryanodine Receptors are the largest ion channels known to date and present major challenges for structural biology. Since their discovery in the 1980s, significant progress has been made in understanding their behaviour through multiple structural methods. Cryo-electron microscopy reconstructions of intact channels depict a mushroom-shaped structure with a large cytoplasmic region that presents many binding sites for regulatory molecules. This region undergoes significant motions during opening and closing of the channel, demonstrating that the Ryanodine Receptor is a bona fide allosteric protein. High-resolution structures through X-ray crystallography and NMR currently cover $\sim 11 \%$ of the entire protein. The combination of high- and low-resolution methods allows us to build pseudo-atomic models. Here we present an overview of the electron microscopy, NMR, and crystallographic analyses of this membrane protein giant.
\end{abstract}

calcium release, excitation-contraction coupling, genetic disease, structural biology, calcium release channel, ion channel

Citation: Kimlicka L, Van Petegem F. The structural biology of ryanodine receptors. Sci China Life Sci, 2011, 54: 712-724, doi: 10.1007/s11427-011-4198-2

Ryanodine Receptors (RyRs) are high-conductance ion channels located in the endoplasmic/sarcoplasmic reticulum (ER/SR). Being permeable to $\mathrm{Ca}^{2+}$, they are involved in various physiological events such as learning and memory, secretion, fertilization, and apoptosis. Their most prominent role is in the contraction of both cardiac and skeletal muscle.

Their name originates from the ability of the channel to bind the plant alkaloid ryanodine at nanomolar affinity, which locks the channel in subconductance states at low concentrations, and blocks them entirely at higher concentrations [1]. RyRs are the largest ion channels currently known, and form a homotetrameric assemblies measuring approximately $2.3 \mathrm{MDa}(565 \mathrm{kD} /$ subunit). In mammalian organisms, three isoforms have been identified, encoded on

*Corresponding author (email: filip.vanpetegem@gmail.com) different chromosomes. RyR1 is the predominant isoform in skeletal muscle [2], while RyR2 is enriched in the heart [3]. The third subtype (RyR3) was originally identified in the brain [4], but all three isoforms are expressed in multiple cell types. They share about $70 \%$ sequence identity, and the sequence deviations mainly stem from three divergent regions in the $R Y R$ genes, referred to as DR1, DR2, and DR3 [5].

RyRs are crucial in excitation-contraction (E-C) coupling. In myocytes, depolarization of the plasma membrane propagates through invaginations in the sarcolemma (transverse tubules or T-tubules), where L-type voltage-gated $\mathrm{Ca}^{2+}$ channels $\left(\mathrm{Ca}_{V} \mathrm{~s}\right)$ are located. The activation of these $\mathrm{Ca}_{V} \mathrm{~s}$ by membrane depolarization stimulates RyRs in the SR. Thus, $\mathrm{Ca}_{\mathrm{V}} \mathrm{s}$ act as the voltage sensor for RyRs [6-8]. In skeletal muscle, RyR1 and $\mathrm{Ca}_{\mathrm{V}} 1.1$ interact directly $[9,10]$, and this mechanical coupling is sufficient for the activation of RyR1. 
On the other hand, in the cardiac muscle, RyR2 is activated by the small influx of extracellular $\mathrm{Ca}^{2+}$ through $\mathrm{Ca}_{\mathrm{V}} \mathrm{S}$ in a process known as $\mathrm{Ca}^{2+}$-induced- $\mathrm{Ca}^{2+}$ release (CICR). In either case, the end result is a massive release of $\mathrm{SR} \mathrm{Ca}^{2+}$ into the myoplasm through RyRs in response to membrane depolarization, leading to muscle contraction. RyRs thus act as signal amplifiers, increasing the $\mathrm{Ca}^{2+}$ message originating from L-type $\mathrm{Ca}_{\mathrm{V}} \mathrm{s}$. The T-tubules and the SR form very close contacts $(\sim 12 \mathrm{~nm})$ in junctional sites [11]. In skeletal muscle, junctional sites are known as triads, while they form dyads in cardiac myocytes [12]. The proximity between the two membranes allows a rapid and precise communication between the voltage sensor and the signal amplifier.

Mutations in RyRs have been linked to severe disease phenotypes, reflecting their importance in the tight regulation of $\mathrm{Ca}^{2+}$ release during muscle contraction [13]. More than 200 mutations have been identified in RyR1, and they are associated with malignant hyperthermia, central core disease, and several other conditions. In the cardiac isoform, more than 150 mutations have been linked to catecholaminergic polymorphic ventricular tachycardia (CPVT) and arrhythmogenic right ventricular dysplasia type 2 (ARVD2), two conditions that can lead to sudden cardiac death $[14,15]$.

The RyR provides a platform for an abundance of small molecules and protein binding partners, and this is the likely reason for the enormous size of this protein [16-21]. These include, but are not limited to, $\mathrm{Ca}^{2+}$, calmodulin, FK506binding proteins (FKBPs), calsequestrin, PKA, and CaMKII. The size of this modulatory complex can be up to $7 \mathrm{MDa}$ [17].

The presence of RyRs in the SR has been observed more than 40 years ago, long before their identification. In electron microscopic images, regularly spaced electron-dense protrusions were observed extending from the SR membrane and spanning the gap in the triads [22]. Not knowing the identity of these structures as $\mathrm{Ca}^{2+}$ release channels at the time, these protrusions were later referred to as "feet" structures [23]. Since then, multiple studies have generated 3-dimensional structures of RyRs, which has provided a wealth of information on the molecular mechanisms underlying these complex channels.

In this review, we focus on the progress of select studies that provide direct structural information on RyRs, including cryo-electron microscopy (cryo-EM), NMR, and X-ray crystallography. Many additional methodologies, such as FRET, have provided key structural information on the RyR architecture, but this is beyond the scope of this review.

\section{Cryo-EM studies of closed RyRs}

Knowing the atomic details of a macromolecular assembly greatly enhances our insights into a particular protein. Unfortunately, RyRs present major challenges to techniques such as X-ray crystallography because of its enormous size, its integral membrane protein nature, and dynamic features that result in a heterogeneity of functional states. For this reason, electron microscopy of single particles and twodimensional crystals has been the primary method of choice in the field for many years.

The presence of junctional "feet" structures has been observed almost half a century ago in thin-section or negative stain electron microscopic images. In these studies, images captured square-shaped, electron-dense protrusions from the SR membrane from both native muscle tissues and isolated $\mathrm{SR}$ vesicles. For a limited set of examples, see references [9,22-28]. The feet were shown to be composed of four subunits [29], and their identity was confirmed when electron microscopic images of purified RyRs were able to show the characteristic "feet" structures [30].

An intriguing property of RyRs is their ability to form two-dimensional arrays. It was observed in native tissue preparations that skeletal muscle RyRs form checkerboard-like lattices, touching the neighbouring molecules near the corners $[11,29,31]$. It was later found that this is an intrinsic property, as purified RyR1 was able to assemble into similar patterns and could form two-dimensional crystals $[32,33]$. In skeletal muscle preparations, tetrads of $\mathrm{Ca}_{\mathrm{V}} \mathrm{s}$ were found to coincide with the cytoplasmic region of RyR 1 in an alternating fashion. This strongly suggested that the two membrane proteins, each being part of different membranes, physically interact [9]. The functional implications of the clustering and RyR1-Ca $a_{V}$ interaction will not be discussed here but are reviewed elsewhere $[6,13,19,34]$.

Numerous efforts have been made to improve the structural details of RyRs in the electron micrographs. Due to its high abundance in the SR membrane and the relative ease of purification, the RyR1 structure has been the most intensively studied. One group obtained the $37 \AA$-resolution structure of RyR1 by generating computer-averaged images from multiple negative stain images $[31,35]$. The resolution was further improved to $\sim 30 \AA$ by the use of frozenhydrated sample preparations in cryo-electron microscopy (cryo-EM) [36-38]. By this time, enough details were present to identify globular-shaped domains in the cytoplasmic region of the channel. Further description was given to the RyR structure: regions found at the corners were termed "clamps" as they resembled the shapes of laboratory clamps, whereas the regions that connected these corners were termed "handles" [38] (Figure 1).

Comparison between the structures of the three isoforms showed that the overall features are universal among the subtypes, which is not surprising given their high sequence homology [39-42]. The most striking trait of the RyR is its mushroom shape, composed of two parts: a larger cytoplasmic region forming the cap $(\sim 80 \%)$ and a smaller transmembrane region forming the stem $(\sim 20 \%)$. Both form square prisms that are rotated by $\sim 40^{\circ}$ relative to one another, and which are connected via four thick columns $\sim 14 \AA$ 


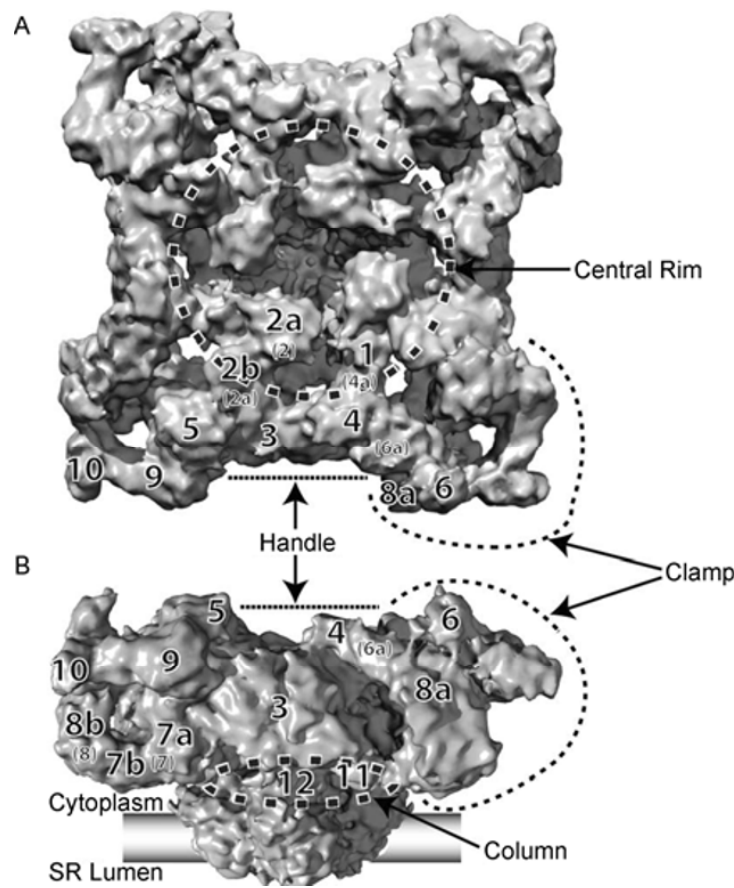

Figure 1 Cryo-EM of closed RyR. The $9.6 \AA$ map (EMDB accession code 1275) of closed RyR1 [43] showing A. a view from the cytoplasm facing the SR and B. from the side in the plane of the SR membrane. Subregions are labelled according to [44], with some alternatives according to [45] shown in brackets. All cryo-EM images, unless mentioned otherwise, were prepared using the software package "UCSF Chimera" [146].

long. The transmembrane region measures $120 \times 120 \times 60$ $\AA^{3}$, whereas the cytoplasmic foot measures approximately $270 \times 270 \times 100 \AA^{3}$. The latter portion contains numerous globular masses and a series of cavities in which more than $50 \%$ of its volume is occupied by solvent. Fifteen globular subregions have been identified so far and arbitrarily assigned. Based on segmentation of the highest resolution map [43,44] (Figure 1), these subregions can be divided into four units: (i) clamps at each corner (subregions 5, 6, 7a, 7b, $8 \mathrm{a}, 8 \mathrm{~b}, 9$, and 10); (ii) handles connecting two clamp regions (subregions 3 and 4); (iii) a central rim on the cytoplasmic face around the fourfold symmetry axis (subregions 1, 2a, and 2b); and (iv) columns (subregions 11 and 12) that connect the cytoplasmic and transmembrane regions. Some of the subregions have received alternative labelling (Figure 1, brackets) [45]. The subunit boundary between each monomer was proposed to center around the handle region, containing one whole handle, two halves of the clamp regions on either side, and a quarter of the central rim [44], although there are some discrepancies which will be discussed in a later section of this review.

The major difference between RyR1 and RyR2 seems to be located in the clamp region, where some masses were found to be either larger or smaller in RyR2 than in RyR1 [39]. Similarly, comparison between RyR1 and RyR3 has revealed extra mass in RyR1 that is missing in RyR3 [42]. These structural differences likely contribute to distinct functional properties among the three isoforms.

A key question surrounding RyR structure is the transmembrane region. How many helices are present, and what is the architecture of the pore-forming part? In order to analyze the transmembrane assembly, the quality and resolution of the maps are of key importance. Two groups have described the structures of closed RyR1 at resolutions near 1 nanometer, with reported resolutions of 10.2-10.3 [45,46] and $9.6 \AA$ [43]. It is noteworthy that both studies used different criteria to report the resolution (Fourier shell correlation or FSC cutoff 0.15 versus 0.5, respectively), adding to the debate of which criterion should be used [47-49].

The highest resolution currently available for any full-length RyR is at $9.6 \AA$ (FSC 0.5) for the closed state of RyR1 [43]. At this resolution, five putative membranespanning $\alpha$ helices in each subunit could be assigned inside rod-like densities. However, there are likely more secondary structure elements, and it has been suggested that each subunit contains 6 or 8 transmembrane helices [50]. Two out of the five putative helices (simply named helices 1 and 2) are located near the 4-fold central axis and resemble the pore-lining and pore helix structures observed in potassium channels (Figure 2). Helix 1 is about $45 \AA$ long and is kinked, bending away from the 4-fold symmetry axis. At the SR luminal side, helices 1 from each subunit together form a large, funnel-like cavity with a diameter of approximately $30 \AA$, which likely forms the channel entrance. The funnel tapers towards the SR membrane, and the narrowest part measures about $15 \AA$ in diameter. Helix 1 is the likely candidate for the inner, pore-lining helix. Helix 2 is shorter $(\sim 22 \AA)$ and is tilted about $50^{\circ}$ with respect to the plane of the SR membrane. Helices 2 from each subunit point towards the 4-fold central axis to form an opening of $\sim 7 \AA$, and are therefore prime candidates for the pore helices. $\mathrm{Pu}$ tative helices 3, 4 and 5 have lengths of about 18-22 A.

Because crystal structures are now available for the pore region of several potassium channels, the helical arrangement can be compared directly with the presumed poreforming region in the cryo-EM reconstructions. Although the $9.6 \AA$ cryo-EM reconstruction is thought to represent RyR 1 in the closed state, helices 1 and 2 only poorly match the helices of the closed KcsA potassium channel struc-

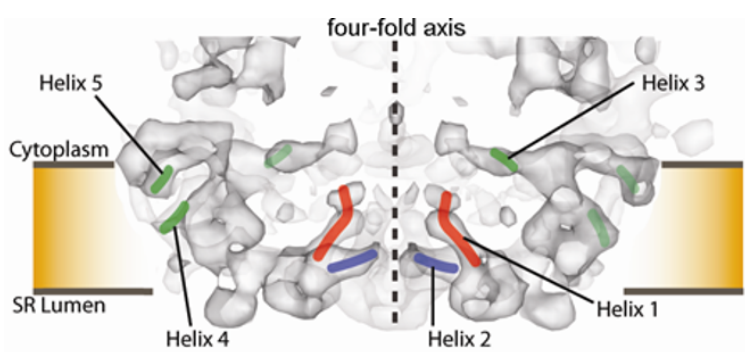

Figure 2 The pore-forming region. Close-up of the transmembrane region in the $9.6 \AA$ closed RyR1 map. The putative helices are outlined. The surrounding density is clipped for clarity. 
ture [51]. Instead, a higher correlation was found for the MthK channel in the open conformation. Kinking of the inner helices in the MthK channel is thought to open this channel [52]. Since helix 1 appears to be bent already, kinking of the inner helices in RyRs is likely not sufficient for channel opening [43]. A proposed model places a highly conserved glycine (G4934) at the hinge position of the inner helix, and two rings of negative charges at the luminal and cytoplasmic openings of the pore. Such a charge distribution is commonly observed in cation-selective channels. For a limited set of examples see [51,53-57].

In contrast with this, different conclusions have been reported by Samso and colleagues. In two RyR1 structures in the closed state at reported resolutions 10.2-10.3 $\AA$ (FSC 0.15 cut-off), manual docking showed a higher correlation between the closed RyR1 and closed KcsA or KirBac1.1 channels $[45,46,51,58]$. Therefore, there are discrepancies in shape of the transmembrane helices and in the interpretations of possible modes of channel gating. These may be due to overall differences in the conformational purity of the samples, or simply differences in resolution $(\sim 4 \AA$ of difference when the FSC 0.5 criterion is applied to all studies). As the structures originated from detergent-solubilized preparations, it cannot be excluded that there are differences depending on sample handling.

More recently, a feature detection program was used to identify secondary structures in the cytoplasmic region using the $9.6 \AA$ structure of RyR1 [44,59]. The authors identified 36 putative $\alpha$ helices and seven putative $\beta$ sheets in the cytoplasmic foot. In particular, one putative $\beta$ sheet is located at subregion 12, near the tapered section of the "column" region. This $\beta$ sheet may play an important role in the gating of the channel since other channels, including Kir and HCN2, share $\beta$ sheets at the same location [58,60-63].

\section{Opening and closing}

Being such a large protein, it is conceivable that opening and closing of the channel is allosterically coupled to the large cytoplasmic foot region. The first open structure of RyR was obtained by Orlova and colleagues at $30 \AA$ resolution [64], followed by more structures in the subsequent years $[42,46,65]$. In these studies, the open conformation of RyR was stabilized by the presence of $\mathrm{Ca}^{2+}$ and other modulators, such as ryanodine, PCB95, and ATP homologs.

The studies all agree that the closed and open RyRs share similar overall structures. One obvious difference is a widening of the central pore upon opening. The authors also reported global conformational changes between the two states, especially in the clamps and in the transmembrane region. Since structural changes occur far away from the proposed pore region, RyRs are clearly allosteric proteins, and interference with any of the movements is likely to affect channel function.
The highest resolution available for the open-state RyR thus far is at 10.2 $\AA$ (FSC 0.143) for RyR1 [46]. The authors used PCB95, a neuroactive compound, to stabilize the open state. The largest conformational changes were found in the cytoplasmic foot. In the open conformation, subregions $7,8,9$, and 10 in the clamp move down towards the SR membrane by $\sim 8 \AA$, while subregion 6 moves outwards by $\sim 5 \AA$ (Figure 3 ). In addition, subregion 2 in the central rim moves upward towards the T-tubule and outwards away from the 4-fold central axis. Thus, the main conformational change in the cytoplasmic portion upon opening of the channel is an outward movement away from the center.

Another large structural change was observed in the transmembrane region. The authors identified at least six membrane-spanning $\alpha$ helices per subunit, based on the number of high density segments in the transmembrane region. Upon opening, the inner helices seem to kink, as the bottom halves shift $3 \AA$ upward towards the cytoplasmic side, and tilt $\sim 5^{\circ}$, widening the pore. This observation is consistent with other studies that reported rotating motions in the transmembrane region and compared the opening and closing of RyR to that of a camera aperture [42,64,65]. The motions in the cytoplasmic and transmembrane regions seem to be coupled via the column region, in particular the inner branches, which tilt by $\sim 8^{\circ}$ upon opening, moving their midpoints $\sim 6 \AA$ away from one another [46].

Manual docking of $\mathrm{K}^{+}$channel structures in the openstate map showed that the open RyR1 correlates better with open $\mathrm{K}^{+}$channels [46]. However, since there have been in-
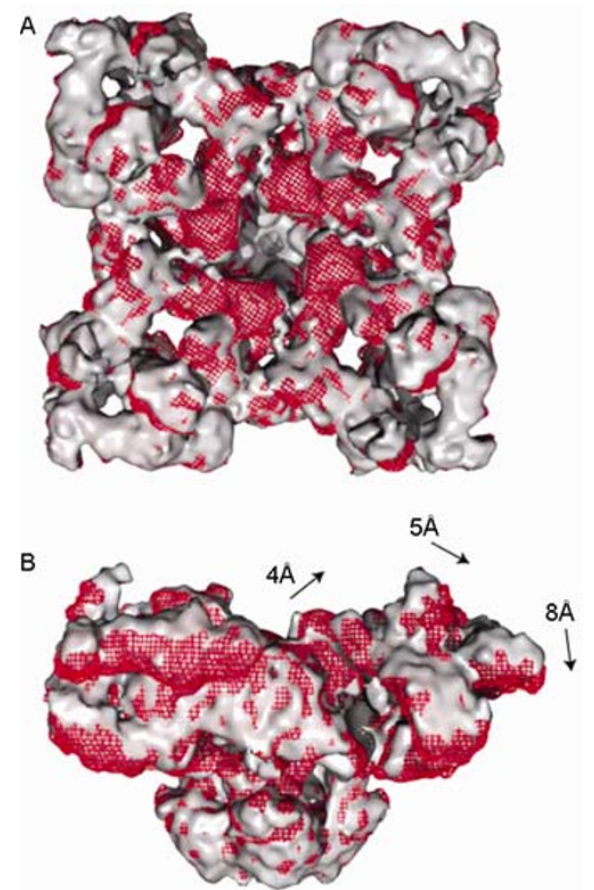

Figure 3 Opening and closing. A and B, Two different views of superpositions of closed (gray, EMDB accession code 1606) and open RyR1 maps (red mesh, EMDB accession code 1607). The extent of some conformational changes is indicated by arrows. 
consistent results for correlation between closed RyR1 and $\mathrm{K}^{+}$channel structures $[43,45,46]$, care must be taken when interpreting these manual docking results. Although buffer conditions can favour an open or closed state in intact membranes, this does not necessarily extrapolate to conditions following detergent extraction. As differences in sample handling may also contribute to structural changes, the conformational state is best deduced by analyzing the final model rather than assuming a particular state. The discrepancies would be reconciled, for example, if the $9.6 \AA$ RyR1 structure [43] represents an open or partially open (i.e. subconducting) channel, rather than a fully closed structure. Future experiments will undoubtedly shed more light on this matter.

\section{Insertion studies}

Despite the continued improvements into the quality of full-length RyR structures, the cryo-EM reconstructions are of insufficient resolution to locate individual amino acids. In the absence of atomic models, one alternative is to determine the rough location of regions of interest via the use of antibodies or fusion proteins. In both cases, analysis of the difference densities found in cryo-EM reconstructions puts restraints on the position of the insertion site or the antibody epitope. The residual uncertainties of the locations depend on the resolution, the size of the insert or antibody, and the lengths of flexible linkers and loops at the insertion site. The locations proposed in many of these studies are summarized in Figure 4A.

The three-dimensional localization of the amino terminus of RyR has been of great interest since the N-terminal re- gion (residues 1-614 in RyR1) covers the first of the three hot spots for disease mutations. The $34 \AA$ cryo-EM reconstruction of RyR3, N-terminally fused to GST (GST-RyR3), displays an extra mass at the clamp region in the middle of subregions 7-10 and a smaller extra mass between subregions 1, 3, and 4 [66]. A $33 \AA$ structure of RyR2 with GFP inserted after Ser 437 presented additional density between subregions 5 and 9 [67]. These studies led the authors to conclude that the $\mathrm{N}$-terminal disease hot spot was most likely located in the clamp region.

Another site of interest in the N-terminal region is residues 590-609 in RyR1 and residues 601-620 in RyR2 which constitute the proposed binding site for dantrolene $[68,69]$. Dantrolene is the only effective treatment for malignant hyperthermia, thus knowing the exact location is of considerable interest. Insertions of GFP in the binding site did not yield any well-behaved protein, but the $27 \AA$ structure of RyR2 with GFP inserted after Tyr846 showed a major extra mass on the T-tubule side of subregion 9. Along with FRET studies, it was concluded that dantrolene also binds close to subregion 9, near the binding site for FKBP12 [70].

The central region (residues 2163-2458 in RyR1) harbours another disease-mutation hot spot. Insertion of GFP near RyR2 Ser2367 showed a significant extra mass in the bridging density between subregions 5 and 6 [71]. This experiment suggests that it is possible for the $\mathrm{N}$-terminal and central disease hot spots to interact, supporting the zipperhypothesis, which states that the two hot spot regions interact and undergo relative conformational changes upon channel opening [72].

The three RyR isoforms share about $70 \%$ identity, and the major sequence differences are located in three stretches
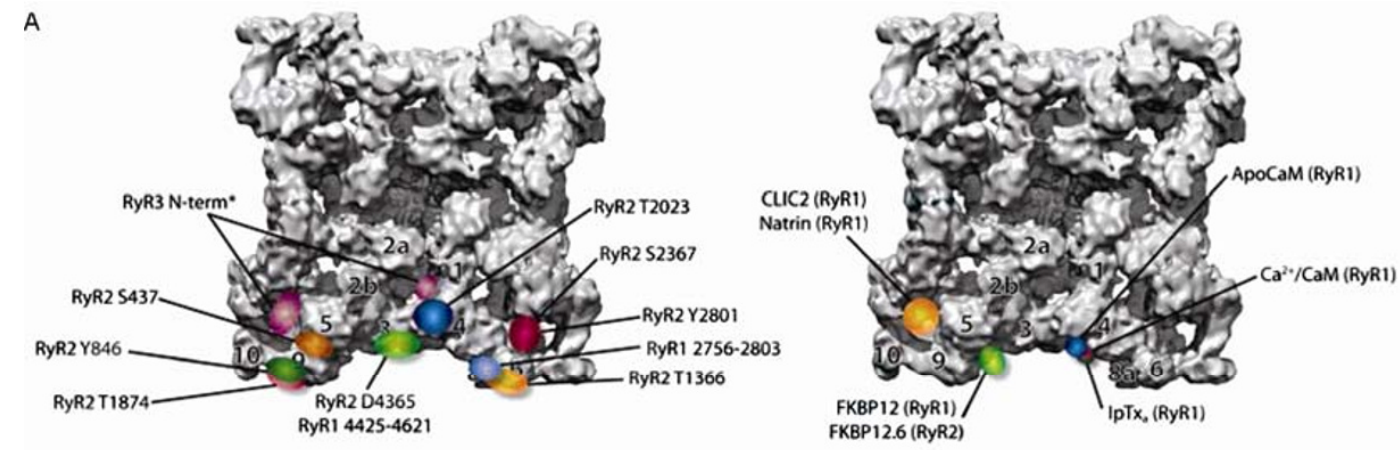

B
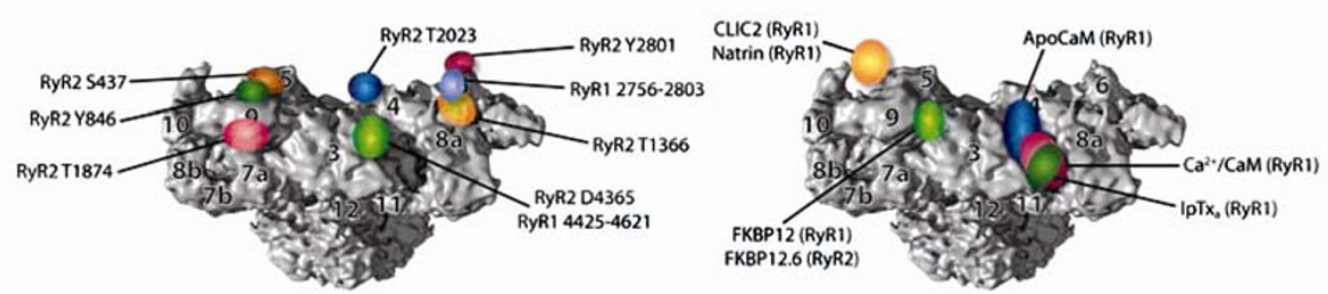

Figure 4 Difference cryo-EM. Overview of difference densities observed for fusion proteins and binding partners. Although these studies were performed on different RyR isoforms, all are shown here on the RyR1 cryo-EM map to allow a direct comparison. A, View from the cytoplasm facing the SR. B, Side view in the plane of the SR membrane. 
known as divergent regions DR1, DR2, and DR3 [5]. These stretches underlie functional differences between the isoforms and their three-dimensional location is therefore valuable for understanding functional mechanisms in RyRs.

DR1 is the largest of the three divergent regions and includes residues 4254-4631 in RyR1 and residues 42104562 in RyR2. This region is thought to be responsible for the varying sensitivities towards $\mathrm{Ca}^{2+}$ inactivation in different isoforms [73-75]. One study took advantage of a monoclonal antibody raised against residues 4425-4621 [76]. The $34 \AA$ structure of RyR1 in complex with the antibody showed an extra mass in subregion 3. In agreement with this observation, an extra mass was observed in the same area after inserting GFP at Asp4365 [77].

The second divergent region, DR2, includes residues 1342-1403 in RyR1 and residues 1353-1397 in RyR2. This region is thought to be involved in E-C coupling [78,79]. Interestingly, DR2 is absent in RyR3 [5]. Negative stain electron micrographs of RyR1 in presence of an antibody against DR2 revealed an extra density in the clamp, close to subregion 9 [41]. Consistent with the result, an extra mass appeared in subregion 6 in a $34 \AA$ structure of RyR2 with GFP inserted after Thr1366 [80].

DR3 includes residues 1872-1923 in RyR1 and residues 1852-1890 in RyR2. This region has been implicated in modulation by $\mathrm{Ca}_{\mathrm{V}} 1.1, \mathrm{Ca}^{2+}$, and FKBP [81-83], and is the target for some disease mutations [84]. Insertion of GFP after RyR2 Thr1874 leads to an extra density in subregion 9, close to the proposed FKBP12.6 binding site [85].

RyRs are the target for phosphorylation by PKA, which is known to activate the channels $[86,87]$. Both Ser2030 and Ser2808 are PKA phosphorylation sites [88-90]. The $27 \AA$ structure of RyR2 with GFP inserted after Thr2023 showed an extra mass in the T-tubule face of subregion 4 [91]. Similarly, the $27 \AA$ structure of RyR2 fused to GFP after Tyr2801 displayed an extra mass in the bridging density connecting subregions 5 and 6 [92]. In agreement, an extra mass appeared in the periphery of subregion 6 in the $25 \AA$ structure of RyR1 in complex with a monoclonal antibody raised against residues 2756-2803 (RyR2 residues 27222769) [92]. One hypothesis concerning the effect of PKA on RyR2 is that it causes the dissociation of FKBP12.6, a protein thought to stabilize the closed state of the channel [93-95]. PKA phosphorylation would thus lead to enhanced channel opening. However, given the large distance between the FKBP12.6 binding site (see section below) and the presumed position of Ser2808, the authors concluded that Ser2808 cannot be directly involved in FKBP12.6 binding.

In the absence of atomic-resolution structures for the majority of the RyR parts, the use of monoclonal antibodies and protein insertions in conjunction with three-dimensional reconstructions continue to provide approximate locations of RyR components. Care must be taken when interpreting the data, as a piece of difference density can also appear further away from the insertion site either due to overall conformational changes or due to the use of long flexible linkers.

\section{The binding sites of protein binding partners}

Using a similar approach, the analysis of difference densities is very useful to locate the position of RyR binding partners (Figure 4B). The differences are either due to the binding partner itself, or due to conformational changes that arise because of the binding. In contrast to fusion protein strategies, such results are not dependent on linker lengths, and the confidence about the exact locations is therefore much higher.

FK506-binding proteins (FKBPs), also known as immunophilins, bind to RyR1 and RyR2 with high affinities. $\mathrm{FKBP} 12$, a $12 \mathrm{kD}$ protein as its name suggests, is found predominantly in skeletal muscle, where it binds to each subunit of RyR1[96]. In the cardiac muscle, FKBP12.6 preferentially associates with RyR2 [97]. In early studies, three-dimensional structures of RyR1-FKBP12 complexes at $\sim 35 \AA$ resolution showed that FKBP12 binds between subregions 3, 5 and 9 [98,99]. In addition, less significant differences were observed in the bridging densities between subregions 5 and 6, which could indicate conformational changes due to the binding [99]. The location was confirmed by a more recent study which showed the structure of the RyR1-FKBP12 complex at $16 \AA$ resolution, and whereby a crystal structure of FKBP12[100] was docked into the difference density [101]. In another recent study, a $33 \AA$ structure of the RyR2-FKBP12.6 complex highlighted a binding site similar to the one for FKBP12 [102]. The binding of FKBP12.6 seemed to cause conformational changes, as subregion 6 was more extended towards the T-tubule and electron dense parts in the transmembrane region splayed apart.

Calmodulin $(\mathrm{CaM})$ binds to RyR1 in both the absence (apoCaM) and presence $\left(\mathrm{Ca}^{2+} / \mathrm{CaM}\right)$ of $\mathrm{Ca}^{2+}$. The binding site of $\mathrm{Ca}^{2+} / \mathrm{CaM}$ on RyR1 was first studied using goldcluster labelled CaM[103] and later confirmed by a $37 \AA$ RyR1-CaM complex [99]. $\mathrm{Ca}^{2+} / \mathrm{CaM}$ binds between subregions 3 and $8 \mathrm{a}$, and its association did not seem to cause major alterations in the conformation of RyR1. The $\mathrm{Ca}^{2+}$ / CaM binding site is approximately $90 \AA$ away from the FKBP12 binding site [99]. Similarly, an RyR1-apoCaM complex at $28 \AA$ resolution showed that the apoCaM binding site is located at the outer surface of subregion 3 , just above the binding site for $\mathrm{Ca}^{2+}-\mathrm{CaM}$ and closer to the T-tubule [104]. These studies suggest that binding of $\mathrm{Ca}^{2+}$ can cause one or both CaM lobes to hop from one binding site to another, which supports the different functional effects that $\mathrm{Ca}^{2+} / \mathrm{CaM}$ and apoCaM have on RyR1 [105-107]. 
Chloride intracellular channel 2 (CLIC-2) is a protein that is widely expressed in skeletal and cardiac muscle of vertebrates [108], and which exist in a soluble and membrane-bound form [109]. This 247-residue protein is homologous to the glutathione-S-transferase (GST) superfamily and was found to inhibit both RyR1 and RyR2 [108,110]. A $25 \AA$ structure of the RyR1-CLIC2 complex showed that it binds between subregions 5 and 6 [110]. Moreover, CLIC- 2 binding seemed to separate subregions 9 and 10, resembling the open conformation despite the fact that the pore appeared to be closed.

Being strictly required in E-C coupling and many other $\mathrm{Ca}^{2+}$-dependent events, it is not surprising that RyRs are the targets of various toxins. Among these is imperatoxin A $\left(\operatorname{IpTx}_{\mathrm{a}}\right)$, a 33-residue peptide derived from scorpion venom that is known to bind to both RyR1 and RyR2 with high affinity, inducing subconductance states [111,112]. Because IpTx $_{\mathrm{a}}$ is small, it was fused N-terminally to biotin and bound to avidin. A $29 \AA$ cryo-EM reconstruction of the resulting complex with RyR1 showed that $\mathrm{IpTx}_{\mathrm{a}}$ binds in the crevice between subregions 3, 7, and 8a [113]. Another toxin known to associate with RyRs is natrin, a 221-residue protein derived from venom of the snake Naja naja atra. Natrin belongs to the cysteine-rich secretory protein (CRISP) family and inhibits RyR1 [114]. A 21 Å resolution structure of the RyR1-natrin complex showed that the toxin binds between subregions 5 and 6 on the surface exposed to the cytoplasm [114].

\section{X-ray crystallography and NMR}

Being giant eukaryotic proteins, RyRs present major challenges to high-resolution studies. The cryo-EM reconstructions have shown that the RyR consists of individual globular regions, which opens up the avenue for high-resolution studies of individual RyR domains. To date, six different studies have described high-resolution structures of individual RyR portions.

\subsection{EF-hand proteins in complex with RyR peptides}

The first high-resolution structures came from a small region (RyR1 residues 3614-3643) in complex with calmodulin $(\mathrm{CaM})$ [115] and S100A1 [116]. CaM is an EF-hand containing protein that can fine-tune the $\mathrm{Ca}^{2+}$-dependent feedback to RyRs. For example, at low cytoplasmic $\mathrm{Ca}^{2+}$ concentrations, apoCaM potentiates opening of RyR1, whereas $\mathrm{Ca}^{2+} / \mathrm{CaM}$ has been found to inhibit the channel [105-107]. In contrast, both apoCaM and $\mathrm{Ca}^{2+} / \mathrm{CaM}$ seem to inhibit RyR2 [117,118]. A $2.0 \AA$ crystal structure of $\mathrm{Ca}^{2+} / \mathrm{CaM}$ in complex with an RyR1 peptide (residues 3614-3643) was solved by Mackenzie and coworkers [115]. The RyR1 peptide forms an $\alpha$ helix, with both lobes of CaM binding in an antiparallel arrangement, whereby the N-lobe binds to the C-terminal half of the peptide, and the C-lobe binds the N-terminal half (Figure 5). The main hydrophobic anchors, Trp3620 and Phe3636, have an unusual 1-17 spacing and the $\mathrm{CaM}$ lobes therefore do not interact with one another. NMR ${ }^{1} \mathrm{H}^{-15} \mathrm{~N}$ residual dipolar couplings (RDCs) suggest that the lobes experience independent domain motions in solution, such that the RDCs for the two lobes cannot be fitted simultaneously to the crystal structure. This is compatible with the notion that multiple CaM binding domains (CaMBDs) are present within the RyR, and that individual lobes may associate with distinct CaMBDs $[119,120]$. Since the cryo-EM showed only one CaM bound per RyR monomer, these stretches have to be close for $\mathrm{CaM}$ to bridge. Given the large number of possible RyR fragments that can bind $\mathrm{CaM}[2,121-123]$, understanding the binding and effects of apoCaM and $\mathrm{Ca}^{2+} / \mathrm{CaM}$ on RyRs involves solving a complex puzzle.

S100A1 is an EF-hand containing protein from the S100 protein family. It exists as homodimers, with each monomer containing a low- and a high-affinity EF hand. It enhances the opening of both RyR1 and RyR2 [124-127]. An NMR structure has shown the interaction between $\mathrm{Ca}^{2+} / \mathrm{S} 100 \mathrm{~A} 1$ and human RyR1 (3616-3627) [116]. Interestingly, some residues (including Trp3620) are involved in binding both $\mathrm{CaM}$ and S100A1, showing that both proteins compete for the same binding site. It is therefore possible that the effect of S100A1 is due to a competition with CaM: at high $\mathrm{Ca}^{2+}$
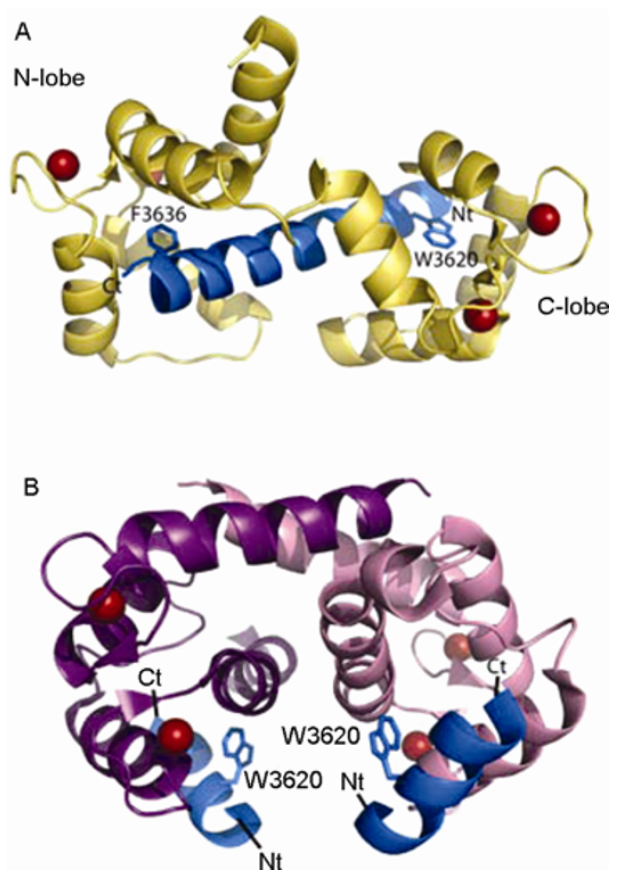

Figure 5 EF hand containing binding partners. A, Crystal structure of $\mathrm{Ca}^{2+} / \mathrm{CaM}$ (yellow) bound to an RyR1 peptide (blue, residues 3614-3643) (PDB accession 2BCX). B, NMR structure of an S100A1 dimer (purple) bound to two RyR1 peptides (blue, residues 3616-3627) (PDB accession $2 \mathrm{~K} 2 \mathrm{~F}$ ). Aromatic anchors providing major contacts are indicated (W3620 and F3636). Red spheres represent $\mathrm{Ca}^{2+}$ ions. All crystal and NMR structure figures were prepared using PyMOL (www.pymol.org). 
levels, S100A1 can compete with $\mathrm{Ca}^{2+} / \mathrm{CaM}$ and therefore abolish its inhibitory effect on the channel.

\subsection{The amino-terminal hot spot}

Both RyR1 and RyR2 have been implicated in a number of severe genetic diseases. All together, over 300 different point mutations have been associated with these disorders [13-15]. Although there may be a significant amount of sequencing bias [128], most mutations are found in three clusters or "hot spots". One of these covers the first $\sim 600$ amino acid residues known as the $\mathrm{N}$-terminal disease hot spot. Several crystallographic studies have focused on this region. The first structure was reported by Ikura and coworkers [129], covering the first domain of RyR1 (residues 1-205). This was soon followed by another study, describing the same domain in both RyR1 and RyR2 [130]. In both studies, the amino-terminal domain (referred to as "domain A" from here on) consists of a $\beta$-trefoil core, built up by twelve $\beta$ strands, and a single $\alpha$ helix (Figure 6). In line with the high degree of sequence conservation, only minor differences are present between domains A of RyR1 and RyR2. Both isoforms contain several disease mutations, many of which are located in a loop connecting strands $\beta 8$ and $\beta 9$, also referred to as a HS (hot spot) loop [129]. Neither NMR nor crystallographic studies of mutant forms of this domain showed any significant differences in stability or structure $[129,130]$. Because the mutations appeared to be at the surface of domain A, it was assumed that they were located at interfaces for other RyR domains or auxiliary proteins.

A recent study has depicted the $2.5 \AA$ crystal structure of the first 559 residues of RyR1, a region that covers the bulk of the N-terminal hot spot [131]. The hot spot folds up as 3 independent domains that interact with one another in a compact form (Figure 6). Domains A and B both fold up as $\beta$-trefoil cores, whereas domain $\mathrm{C}$ consists of a bundle of 5 $\alpha$-helices. The domain-domain interactions are mainly hydrophilic in nature and inherently weak. In RyR1 and RyR2 combined, up to 56 different disease mutations could be located on the structure (Figure 6). Fifteen of those are located at the interface between domains A, B, and C, and seem to destabilize the domain-domain interactions. These include mutations that abolish ionic pairs across the domains. Six mutations are buried within individual domains, likely causing local misfolding or generally destabilizing the protein. All other mutations are found at the periphery of the crystal structure.

A very severe form of CPVT is due to the deletion of a 35-residue segment (exon 3) in the $\mathrm{N}$-terminal domain of RyR2. The deletion ablates an $\alpha$ helix and a $\beta$ strand that is part of the domain A $\beta$-trefoil core, but unexpectedly does not cause misfolding. Even more surprising is that the deletion enhances the thermal stability of the domain [130]. A
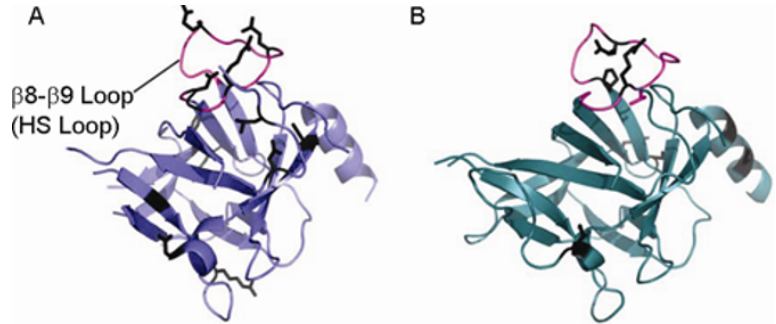

C

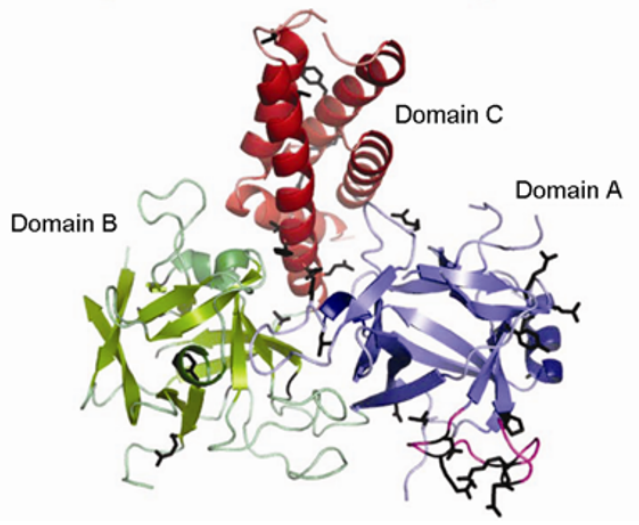

Figure 6 The N-terminal disease hot spot. Crystal structures of RyR1 domain A (PDB accession 3HSM) (A), highlighting the hot spot (HS) loop (purple), RyR2 domain A (PDB accession 3IM5) (B) and RyR1 domains A, blue; B, green; C, red (PDB accession 2XOA) (C). The positions of amino acid residues involved in disease (RyR1 and RyR2 combined) are shown in black.

recent crystal structure of RyR2 domain A with exon 3 deleted highlights a very unusual 'structural rescue', whereby a flexible loop (exon 4), unique to RyR2, takes over the position of the deleted $\beta$ strand [132]. This observation suggests that alternative splicing, allowing either exon 3 or exon 4 to insert itself in the $\beta$-trefoil core, allows for finetuning the activity of RyR2.

\section{Pseudo-atomic models}

Cryo-EM images of the entire receptor currently cannot locate the positions of individual amino acids. Concurrently, high-resolution structures of individual portions provide limited information when their locations in the full-length proteins are unknown. However, with both techniques combined, it is feasible to start constructing pseudo-atomic models, by docking high-resolution fragments into cryo-EM maps.

Several algorithms exist to dock fragments, making use of six-dimensional searches (three translational and three rotational parameters) along with a scoring function. Crucial parameters for a successful experiment include the quality and resolution of the cryo-EM reconstruction, and the relative size of the fragment compared to the intact protein. When only small fragments are used, a simple crosscorrelation coefficient may not suffice to provide a reliable position [133,134]. In those cases, it may be advantageous 
to make use of a Laplacian filter, which enhances the features of the surface. Although the latter may increase highfrequency noise, the ideal method for any particular case can be identified through a statistical analysis of the correlation coefficients, whereby a correct solution has a correlation coefficient that is significantly higher than the second best solution. In the absence of such a docking contrast, the reliability of the top solution may be questionable.

Prior to the availability of high-resolution structures, some homology-based models were prepared for the $\mathrm{N}$ terminal region, and these were docked in the clamp region $[44,135]$. However, using the crystal structure of the $\mathrm{N}$-terminal disease hot spot, a high-contrast solution places the N-terminal disease hot spot in the central rim, forming a vestibule around the fourfold symmetry axis (Figure 7) [131]. Using the segmentation described by [44], domain A occupies subregion 1 , whereas domains $\mathrm{B}$ and $\mathrm{C}$ occupy subregions $2 \mathrm{a}$ and $2 \mathrm{~b}$, respectively. The different results with the prior docking studies are most likely due to discrepancies between the homology based models and the crystal structure, and due to the use of different docking algorithms. The docking is also at odds with the proposed subunit boundaries [44].

The hot spot thus seems to form interactions with the same region in a neighbouring subunit, mostly involving contacts between domains $\mathrm{A}$ and $\mathrm{B}$. This interface contains the largest concentration of disease mutations (19 so far). A side view shows that the hot spot is located far away from the transmembrane region, but is connected to the poreforming region via electron dense columns (Figure 7). All mutations at the periphery of the disease hot spot were found to be located at one of six different interfaces with other RyR domains. No mutation was found to be located in the exposed surface area, consistent with the fact that no protein binding partner has been found in this region.

A

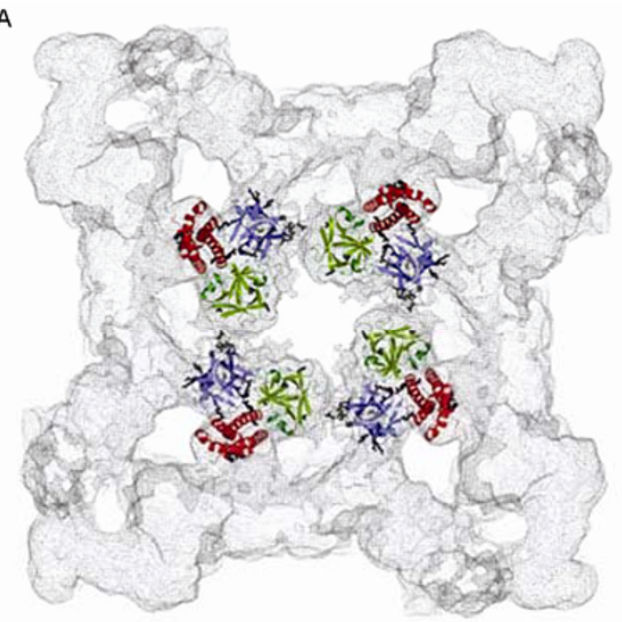

The location of the hot spot shows apparent discrepancies with the cryo-EM analysis of fusion proteins containing GFP inserted near residue Ser437 in RyR2 [67], or with GST fused to the N-terminus of RyR3 [66]. However, when the length of the linkers is taken into account, together with the inherent length of the loop region containing Ser437, these studies can be reconciled [131]. In addition, the GST-RyR3 fusion construct displayed two main regions of difference density, suggesting the fusion created conformational changes. One of these is located right next to the N-terminus of the docked RyR1ABC domains.

The cryo-EM studies comparing RyR1 in the open and closed states have shown significant movements in the central rim where the disease hot spot is located [46]. Together with the distribution of the disease mutations, it is plausible to assume that most or all of the domain-domain interfaces involving the hot spot are labile and subject to movement. This creates an energetic penalty to open the channel, therefore assigning the role of a 'brake' to the hot spot. When disease mutations perturb any of these interfaces, the energetic penalty is reduced, making it easier for the channel to open. This is consistent with the fact that most mutations characterized so far lead to a gain of function, including increased sensitivity to modulators and leakage of $\mathrm{Ca}^{2+}[13]$. Combining the mutations with another event (increased temperature, binding of halogenated anaesthetics, phosphorylation, etc.) is then sufficient to open the channels.

\section{Analogies with the inositol-1,4,5-trisphosphate receptor $\left(\mathbf{I P}_{\mathbf{3}} \mathbf{R}\right)$}

Inositol-1,4,5-trisphosphate receptors $\left(\mathrm{IP}_{3} \mathrm{Rs}\right)$ are $\mathrm{Ca}^{2+}$ release channels, predominantly present in the ER membrane.

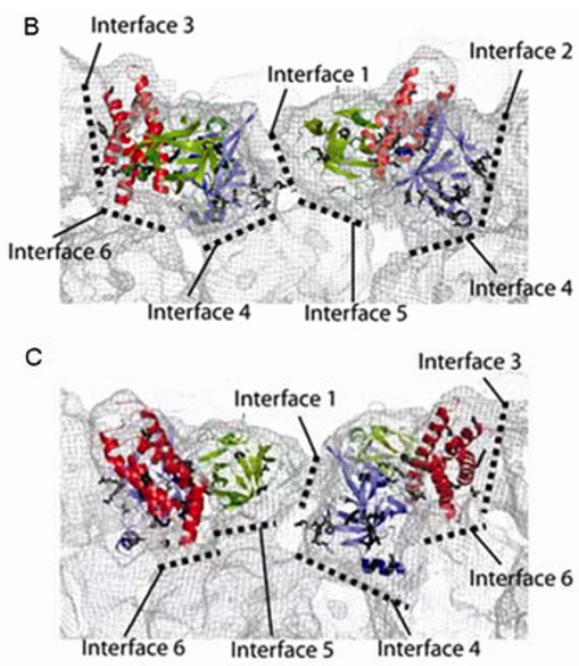

Figure 7 Pseudo-atomic models. Top view (A) and two side views ( $90^{\circ}$ rotation) (B and C) of the N-terminal disease hot spot docked in the $9.6 \AA$ closedstate cryo-EM map. Disease mutations are shown in black, and interfaces with neighbouring RyR domains are labelled. Note: disease mutations in flexible loops, mainly located at interface 1 , are not shown for clarity. 


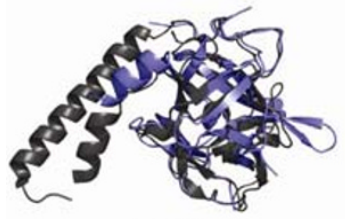

Domain A
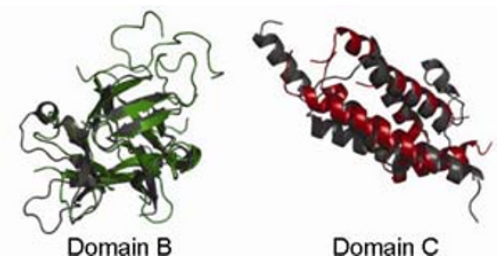

Domain C
Figure 8 Structural conservation. Superposition of domains A, B, and C of RyR1 (PDB accession 2XOA, colored) and $\mathrm{IP}_{3} \mathrm{R} 1$ (PDB accession $1 \mathrm{XZZ}$ and $1 \mathrm{~N} 4 \mathrm{~K}$, gray).

With sizes $\sim 1 \mathrm{MDa}$, the $\mathrm{IP}_{3} \mathrm{R}$ is the "little brother" of the RyRs, and a significant amount of homology ( $30 \%$ sequence identity) is found for both channels. Not surprisingly, a great deal of structural homology is present as well, and $\mathrm{IP}_{3} \mathrm{Rs}$ have been studied by both electron microscopy and $\mathrm{X}$-ray crystallography. Several cryo-EM reconstructions of $\mathrm{IP}_{3} \mathrm{Rs}$ have been performed, but since most seem at odds with one another, it is difficult at this point to directly compare these with the RyR reconstructions [136-141].

Three $\mathrm{IP}_{3} \mathrm{R}$ domains have been investigated with X-ray crystallography. Crystal structures are available for the $\mathrm{N}$-terminal suppressor domain (equivalent to domain $\mathrm{A}$ ) $[142,143]$, and for the following two domains (B and C, together known as the $\mathrm{IP}_{3}$ binding core) in complex with $\mathrm{IP}_{3}$ [144]. However, a structure describing all three domains together has thus far remained elusive. The RyR1 ABC domains have a surprisingly large amount of structural homology with the $\mathrm{IP}_{3} \mathrm{R}$ N-terminal domains, a feature that was predicted before the RyR 1 ABC crystal structure was determined [44]. The secondary structure elements in the two $\beta$-trefoil domains and the $\alpha$-helical bundle in domain $\mathrm{C}$ superpose very well (Figure 8) [145]. The structural conservation underlines the evolutionary relationship between both channels, and suggests that the interactions between the suppressor domain and the $\mathrm{IP}_{3}$ binding core may be very similar as well $[131,145]$.

\section{Conclusion}

The ryanodine receptor is a huge membrane protein for which high-resolution has only recently become available. Despite a number of studies, only $\sim 11 \%$ of the channel has been covered at high resolution. Many additional studies remain to be done, either filling out all pieces of the three-dimensional puzzle with pseudo-atomic models, or perhaps a high-resolution structure of the entire edifice. To be continued...

We thank Kelvin Lau for comments on our manuscript. FVP is funded by the CIHR (operating grant 84350) and the Heart and Stroke Foundation of Canada. FVP is a CIHR new investigator and a Michael Smith Foundation for Health Research Scholar.

1 Meissner G. Ryanodine activation and inhibition of the $\mathrm{Ca}^{2+}$ release channel of sarcoplasmic reticulum. J Biol Chem, 1986, 261: 6300-6306

2 Takeshima H, Nishimura S, Matsumoto T, et al. Primary structure and expression from complementary DNA of skeletal muscle ryanodine receptor. Nature, 1989, 339: 439-445

3 Otsu K, Willard H F, Khanna V K, et al. Molecular cloning of cDNA encoding the $\mathrm{Ca}^{2+}$ release channel (ryanodine receptor) of rabbit cardiac muscle sarcoplasmic reticulum. J Biol Chem, 1990, 265: 13472-13483

4 Hakamata Y, Nakai J, Takeshima H, et al. Primary structure and distribution of a novel ryanodine receptor/calcium release channel from rabbit brain. FEBS Lett, 1992, 312: 229-235

5 Sorrentino V, Volpe P. Ryanodine receptors: How many, where and why? Trends Pharmacol Sci, 1993, 14: 98-103

6 Endo M. Calcium-induced calcium release in skeletal muscle. Physiol Rev, 2009, 89: 1153-1176

7 Rios E, Brum G. Involvement of dihydropyridine receptors in excitation-contraction coupling in skeletal muscle. Nature, 1987, 325: 717-720

8 Tanabe T, Beam K G, Powell J A, et al. Restoration of excitationcontraction coupling and slow calcium current in dysgenic muscle by dihydropyridine receptor complementary DNA. Nature, 1988, 336: 134-139

9 Block B A, Imagawa T, Campbell K P, et al. Structural evidence for direct interaction between the molecular components of the transverse tubule/sarcoplasmic reticulum junction in skeletal muscle. J Cell Biol, 1988, 107: 2587-2600

10 Tanabe T, Beam K G, Adams B A, et al. Regions of the skeletal muscle dihydropyridine receptor critical for excitation-contraction coupling. Nature, 1990, 346: 567-569

11 Saito A, Seiler S, Chu A, et al. Preparation and morphology of sarcoplasmic reticulum terminal cisternae from rabbit skeletal muscle. J Cell Biol, 1984, 99: 875-885

12 Smith D S. The organization and function of the sarcoplasmic reticulum and T-system of muscle cells. Prog Biophys Mol Biol, 1966, 16: 107-142

13 Betzenhauser M J, Marks A R. Ryanodine receptor channelopathies. Pflugers Arch, 2010, 460: 467-480

14 Thomas N L, Maxwell C, Mukherjee S, et al. Ryanodine receptor mutations in arrhythmia: The continuing mystery of channel dysfunction. FEBS Lett, 2010, 584: 2153-2160

15 Priori S G, Chen S R. Inherited dysfunction of sarcoplasmic reticulum $\mathrm{Ca}^{2+}$ handling and arrhythmogenesis. Circ Res, 2011, 108: 871-883

16 Kushnir A, Marks A R. The ryanodine receptor in cardiac physiology and disease. Adv Pharmacol, 2010, 59: 1-30

17 Bers D M. Macromolecular complexes regulating cardiac ryanodine receptor function. J Mol Cell Cardiol, 2004, 37: 417-429

18 Zalk R, Lehnart S E, Marks A R. Modulation of the ryanodine receptor and intracellular calcium. Annu Rev Biochem, 2007, 76: 367-385

19 Lanner J T, Georgiou D K, Joshi A D, et al. Ryanodine receptors: structure, expression, molecular details, and function in calcium release. Cold Spring Harb Perspect Biol, 2010, 2: a003996

20 Marx S. Ion channel macromolecular complexes in the heart. J Mol Cell Cardiol, 2003, 35: 37-44

21 Fill M, Copello J A. Ryanodine receptor calcium release channels. Physiol Rev, 2002, 82: 893-922

22 Revel J P. The sarcoplasmic reticulum of the bat cricothroid muscle. J Cell Biol, 1962, 12: 571-588

23 Franzini-Armstrong C. Studies of the triad : I. Structure of the junction in frog twitch fibers. J Cell Biol, 1970, 47: 488-499

24 Bonilla E. Staining of transverse tubular system of skeletal muscle by tannic acid-glutaraldehyde fixation. J Ultrastruct Res, 1977, 162-165

25 Somlyo A V. Bridging structures spanning the junctioning gap at the triad of skeletal muscle. J Cell Biol, 1979, 80: 743-750

26 Campbell K P, Franzini-Armstrong C, Shamoo A E. Further characterization of light and heavy sarcoplasmic reticulum vesicles. Identification of the 'sarcoplasmic reticulum feet' associated with 
heavy sarcoplasmic reticulum vesicles. Biochim Biophys Acta, 1980, 602: $97-116$

27 Franzini-Armstrong C. Membrane particles and transmission at the triad. Fed Proc, 1975, 34: 1382-1389

28 Franzini-Armstrong C. Structure of sarcoplasmic reticulum. Fed Proc, 1980, 39: 2403-2409

29 Ferguson D G, Schwartz H W, Franzini-Armstrong C. Subunit structure of junctional feet in triads of skeletal muscle: A freezedrying, rotary-shadowing study. J Cell Biol, 1984, 99: 1735-1742

30 Inui M, Saito A, Fleischer S. Purification of the ryanodine receptor and identity with feet structures of junctional terminal cisternae of sarcoplasmic reticulum from fast skeletal muscle. J Biol Chem, 1987, 262: $1740-1747$

31 Saito A, Inui M, Radermacher M, et al. Ultrastructure of the calcium release channel of sarcoplasmic reticulum. J Cell Biol, 1988, 107: 211-219

32 Yin $\mathrm{C} \mathrm{C}$, Lai $\mathrm{F}$ A. Intrinsic lattice formation by the ryanodine receptor calcium-release channel. Nat Cell Biol, 2000, 2: 669-671

33 Yin C C, Han H, Wei R, et al. Two-dimensional crystallization of the ryanodine receptor $\mathrm{Ca}^{2+}$ release channel on lipid membranes. J Struct Biol, 2005, 149: 219-224

34 Cheng H, Lederer W J. Calcium sparks. Physiol Rev, 2008, 88: 1491-1545

35 Wagenknecht $\mathrm{T}$, Grassucci R, Frank J, et al. Three-dimensional architecture of the calcium channel/foot structure of sarcoplasmic reticulum. Nature, 1989, 338: 167-170

36 Radermacher M, Rao V, Grassucci R, et al. Cryo-electron microscopy and three-dimensional reconstruction of the calcium release channel/ryanodine receptor from skeletal muscle. J Cell Biol, 1994, 127: 411-423

37 Radermacher M, Wagenknecht T, Grassucci R, et al. Cryo-EM of the native structure of the calcium release channel/ryanodine receptor from sarcoplasmic reticulum. Biophys J, 1992, 61: 936-940

38 Serysheva, II, Orlova E V, Chiu W, et al. Electron cryomicroscopy and angular reconstitution used to visualize the skeletal muscle calcium release channel. Nat Struct Biol, 1995, 2: 18-24

39 Sharma M R, Penczek P, Grassucci R, et al. Cryoelectron microscopy and image analysis of the cardiac ryanodine receptor. J Biol Chem, 1998, 273: 18429-18434

40 Jeyakumar L H, Copello J A, O'Malley A M, et al. Purification and characterization of ryanodine receptor 3 from mammalian tissue. $\mathrm{J}$ Biol Chem, 1998, 273: 16011-16020

41 Murayama T, Oba T, Katayama E, et al. Further characterization of the type 3 ryanodine receptor (RyR3) purified from rabbit diaphragm. J Biol Chem, 1999, 274: 17297-17308

42 Sharma M R, Jeyakumar L H, Fleischer S, et al. Three-dimensional structure of ryanodine receptor isoform three in two conformational states as visualized by cryo-electron microscopy. J Biol Chem, 2000, 275: 9485-9491

43 Ludtke S J, Serysheva II, Hamilton S L, et al. The pore structure of the closed RyR1 channel. Structure, 2005, 13: 1203-1211

44 Serysheva II, Ludtke S J, Baker M L, et al. Subnanometer-resolution electron cryomicroscopy-based domain models for the cytoplasmic region of skeletal muscle RyR channel. Proc Natl Acad Sci USA, 2008, 105: 9610-9615

45 Samso $\mathrm{M}$, Wagenknecht $\mathrm{T}$, Allen $\mathrm{P}$ D. Internal structure and visualization of transmembrane domains of the RyR 1 calcium release channel by cryo-EM. Nat Struct Mol Biol, 2005, 12: 539-544

46 Samso M, Feng W, Pessah I N, et al. Coordinated movement of cytoplasmic and transmembrane domains of RyR1 upon gating. PLoS Biol, 2009, 7: e85

47 Minor D L Jr. Bend to open? Structure, 2005, 13: 1094-1095

48 Frank J. Single-particle imaging of macromolecules by cryo-electron microscopy. Annu Rev Biophys Biomol Struct, 2002, 31: 303-319

49 van Heel M, Schatz M. Fourier shell correlation threshold criteria. J Struct Biol, 2005, 151: 250-262

50 Du G G, Avila G, Sharma P, et al. Role of the sequence surrounding predicted transmembrane helix M4 in membrane association and function of the $\mathrm{Ca}(2+)$ release channel of skeletal muscle sarcoplasmic reticulum (ryanodine receptor isoform 1). J Biol Chem, 2004, 279: 37566-37574

51 Doyle D A, Morais Cabral J, Pfuetzner R A, et al. The structure of the potassium channel: molecular basis of $\mathrm{K}^{+}$conduction and selectivity. Science, 1998, 280: 69-77

52 Jiang Y, Lee A, Chen J, et al. The open pore conformation of potassium channels. Nature, 2002, 417: 523-526

53 D'Avanzo N, Cho H C, Tolokh I, et al. Conduction through the inward rectifier potassium channel, Kir2.1, is increased by negatively charged extracellular residues. J Gen Physiol, 2005, 125: 493-503

54 Imoto K, Busch C, Sakmann B, et al. Rings of negatively charged amino acids determine the acetylcholine receptor channel conductance. Nature, 1988, 335: 645-648

55 Andersen C, Koronakis E, Hughes C, et al. An aspartate ring at the TolC tunnel entrance determines ion selectivity and presents a target for blocking by large cations. Mol Microbiol, 2002, 44: 1131-1139

56 Terlau H, Heinemann S H, Stuhmer W, et al. Mapping the site of block by tetrodotoxin and saxitoxin of sodium channel II. FEBS Lett, 1991, 293: 93-96

57 Long S B, Campbell E B, Mackinnon R. Voltage sensor of Kv1.2: structural basis of electromechanical coupling. Science, 2005, 309: 903-908

58 Kuo A, Gulbis J M, Antcliff J F, et al. Crystal structure of the potassium channel KirBac1.1 in the closed state. Science, 2003, 300: 1922-1926

59 Baker M L, Ju T, Chiu W. Identification of secondary structure elements in intermediate-resolution density maps. Structure, 2007, 15: $7-19$

60 Nishida M, Cadene M, Chait B T, et al. Crystal structure of a Kir3.1prokaryotic Kir channel chimera. EMBO J, 2007, 26: 4005-4015

61 Nishida M, MacKinnon R. Structural basis of inward rectification: cytoplasmic pore of the $\mathrm{G}$ protein-gated inward rectifier GIRK1 at 1.8 A resolution. Cell, 2002, 111: 957-965

62 Pegan S, Arrabit C, Zhou W, et al. Cytoplasmic domain structures of Kir2.1 and Kir3.1 show sites for modulating gating and rectification. Nat Neurosci, 2005, 8: 279-287

63 Zagotta W N, Olivier N B, Black K D, et al. Structural basis for modulation and agonist specificity of HCN pacemaker channels. Nature, 2003, 425: 200-205

64 Orlova E V, Serysheva II, van Heel M, et al. Two structural configurations of the skeletal muscle calcium release channel. Nat Struct Biol, 1996, 3: 547-552

65 Serysheva II, Schatz M, van Heel M, et al. Structure of the skeletal muscle calcium release channel activated with $\mathrm{Ca}^{2+}$ and AMP-PCP. Biophys J, 1999, 77: 1936-1944

66 Liu Z, Zhang J, Sharma M R, et al. Three-dimensional reconstruction of the recombinant type 3 ryanodine receptor and localization of its amino terminus. Proc Natl Acad Sci USA, 2001, 98: 6104-6109

67 Wang R, Chen W, Cai S, et al. Localization of an $\mathrm{NH}(2)$-terminal disease-causing mutation hot spot to the "clamp" region in the threedimensional structure of the cardiac ryanodine receptor. J Biol Chem, 2007, 282: 17785-17793

68 Paul-Pletzer K, Yamamoto T, Bhat M B, et al. Identification of a dantrolene-binding sequence on the skeletal muscle ryanodine receptor. J Biol Chem, 2002, 277: 34918-34923

69 Paul-Pletzer K, Yamamoto T, Ikemoto N, et al. Probing a putative dantrolene-binding site on the cardiac ryanodine receptor. Biochem $\mathrm{J}$, 2005, 387: 905-909

70 Wang R, Zhong X, Meng X, et al. Localization of the dantrolenebinding sequence near the FK506-binding protein-binding site in the three-dimensional structure of the ryanodine receptor. J Biol Chem, 2011, 286: 12202-12212

71 Liu Z, Wang R, Zhang J, et al. Localization of a disease-associated mutation site in the three-dimensional structure of the cardiac muscle ryanodine receptor. J Biol Chem, 2005, 280: 37941-37947

72 Ikemoto N, Yamamoto T. Regulation of calcium release by interdomain interaction within ryanodine receptors. Front Biosci, 2002, 7: d671-683

73 Du G G, Khanna V K, MacLennan D H. Mutation of divergent 
region 1 alters caffeine and $\mathrm{Ca}^{2+}$ sensitivity of the skeletal muscle $\mathrm{Ca}^{2+}$ release channel (ryanodine receptor). J Biol Chem, 2000, 275: $11778-11783$

74 Du G G, MacLennan D H. $\mathrm{Ca}^{2+}$ inactivation sites are located in the $\mathrm{COOH}$-terminal quarter of recombinant rabbit skeletal muscle $\mathrm{Ca}^{2+}$ release channels (ryanodine receptors). J Biol Chem, 1999, 274: 26120-26126

75 Nakai J, Gao L, Xu L, et al. Evidence for a role of C-terminus in $\mathrm{Ca}^{2+}$ inactivation of skeletal muscle $\mathrm{Ca}^{2+}$ release channel (ryanodine receptor). FEBS Lett, 1999, 459: 154-158

76 Benacquista B L, Sharma M R, Samso M, et al. Amino acid residues 4425-4621 localized on the three-dimensional structure of the skeletal muscle ryanodine receptor. Biophys J, 2000, 78: 1349-1358

77 Liu Z, Zhang J, Li P, et al. Three-dimensional reconstruction of the recombinant type 2 ryanodine receptor and localization of its divergent region 1. J Biol Chem, 2002, 277: 46712-46719

78 Yamazawa $\mathrm{T}$, Takeshima $\mathrm{H}$, Shimuta $\mathrm{M}$, et al. A region of the ryanodine receptor critical for excitation-contraction coupling in skeletal muscle. J Biol Chem, 1997, 272: 8161-8164

79 Perez C F, Mukherjee S, Allen P D. Amino acids 1-1,680 of ryanodine receptor type 1 hold critical determinants of skeletal type for excitation-contraction coupling. Role of divergence domain D2. J Biol Chem, 2003, 278: 39644-39652

80 Liu Z, Zhang J, Wang R, et al. Location of divergent region 2 on the three-dimensional structure of cardiac muscle ryanodine receptor/ calcium release channel. J Mol Biol, 2004, 338: 533-545

81 Hayek S M, Zhao J, Bhat M, et al. A negatively charged region of the skeletal muscle ryanodine receptor is involved in $\mathrm{Ca}^{2+}$-dependent regulation of the $\mathrm{Ca}^{2+}$ release channel. FEBS Lett, 1999, 461: 157164

82 Hayek S M, Zhu X, Bhat M B, et al. Characterization of a calcium-regulation domain of the skeletal-muscle ryanodine receptor. Biochem J, 2000, 351: 57-65

83 Proenza C, O'Brien J, Nakai J, et al. Identification of a region of RyR1 that participates in allosteric coupling with the alpha(1S) (Ca(V)1.1) II-III loop. J Biol Chem, 2002, 277: 6530-6535

84 Koop A, Goldmann P, Chen S R, et al. ARVC-related mutations in divergent region 3 alter functional properties of the cardiac ryanodine receptor. Biophys J, 2008, 94: 4668-4677

85 Zhang J, Liu Z, Masumiya H, et al. Three-dimensional localization of divergent region 3 of the ryanodine receptor to the clamp-shaped structures adjacent to the FKBP binding sites. J Biol Chem, 2003, 278: 14211-14218

86 Danila C I, Hamilton S L. Phosphorylation of ryanodine receptors. Biol Res, 2004, 37: 521-525

87 Petrovic M M, Vales K, Putnikovic B, et al. Ryanodine receptors, voltage-gated calcium channels and their relationship with protein kinase A in the myocardium. Physiol Res, 2008, 57: 141-149

88 Wehrens X H, Lehnart S E, Reiken S, et al. Ryanodine receptor/ calcium release channel PKA phosphorylation: A critical mediator of heart failure progression. Proc Natl Acad Sci USA, 2006, 103: $511-518$

89 Xiao B, Jiang M T, Zhao M, et al. Characterization of a novel PKA phosphorylation site, serine-2030, reveals no PKA hyperphosphorylation of the cardiac ryanodine receptor in canine heart failure. Circ Res, 2005, 96: 847-855

90 Xiao B, Zhong G, Obayashi M, et al. Ser-2030, but not Ser-2808, is the major phosphorylation site in cardiac ryanodine receptors responding to protein kinase $\mathrm{A}$ activation upon beta-adrenergic stimulation in normal and failing hearts. Biochem J, 2006, 396: 7-16

91 Jones P P, Meng X, Xiao B, et al. Localization of PKA phosphorylation site, $\operatorname{Ser}(2030)$, in the three-dimensional structure of cardiac ryanodine receptor. Biochem J, 2008, 410: 261-270

92 Meng X, Xiao B, Cai S, et al. Three-dimensional localization of serine 2808, a phosphorylation site in cardiac ryanodine receptor. $\mathrm{J}$ Biol Chem, 2007, 282: 25929-25939

93 Ahern G P, Junankar P R, Dulhunty A F. Subconductance states in single-channel activity of skeletal muscle ryanodine receptors after removal of FKBP12. Biophys J, 1997, 72: 146-162
94 Chelu M G, Danila C I, Gilman C P, et al. Regulation of ryanodine receptors by FK506 binding proteins. Trends Cardiovasc Med, 2004, 14: $227-234$

95 Marx S O, Reiken S, Hisamatsu Y, et al. PKA phosphorylation dissociates FKBP12.6 from the calcium release channel (ryanodine receptor): defective regulation in failing hearts. Cell, 2000, 101: 365-376

96 Jayaraman T, Brillantes A M, Timerman A P, et al. FK506 binding protein associated with the calcium release channel (ryanodine receptor). J Biol Chem, 1992, 267: 9474-9477

97 Timerman A P, Onoue H, Xin H B, et al. Selective binding of FKBP12.6 by the cardiac ryanodine receptor. J Biol Chem, 1996, 271: 20385-20391

98 Wagenknecht $\mathrm{T}$, Grassucci R, Berkowitz J, et al. Cryoelectron microscopy resolves FK506-binding protein sites on the skeletal muscle ryanodine receptor. Biophys J, 1996, 70: 1709-1715

99 Wagenknecht T, Radermacher M, Grassucci R, et al. Locations of calmodulin and FK506-binding protein on the three-dimensional architecture of the skeletal muscle ryanodine receptor. J Biol Chem, 1997, 272: 32463-32471

100 Burkhard P, Taylor P, Walkinshaw M D. X-ray structures of small ligand-FKBP complexes provide an estimate for hydrophobic interaction energies. J Mol Biol, 2000, 295: 953-962

101 Samso M, Shen X, Allen P D. Structural characterization of the RyR1-FKBP12 interaction. J Mol Biol, 2006, 356: 917-927

102 Sharma M R, Jeyakumar L H, Fleischer S, et al. Three-dimensional visualization of FKBP12.6 binding to an open conformation of cardiac ryanodine receptor. Biophys J, 2006, 90: 164-172

103 Wagenknecht T, Berkowitz J, Grassucci R, et al. Localization of calmodulin binding sites on the ryanodine receptor from skeletal muscle by electron microscopy. Biophys J, 1994, 67: 2286-2295

104 Samso M, Wagenknecht T. Apocalmodulin and $\mathrm{Ca}^{2+}$-calmodulin bind to neighboring locations on the ryanodine receptor. J Biol Chem, 2002, 277: 1349-1353

105 Ikemoto T, Iino M, Endo M. Enhancing effect of calmodulin on $\mathrm{Ca}^{2+}$-induced $\mathrm{Ca}^{2+}$ release in the sarcoplasmic reticulum of rabbit skeletal muscle fibres. J Physiol, 1995, 487 ( Pt 3): 573-582

106 Tripathy A, Xu L, Mann G, et al. Calmodulin activation and inhibition of skeletal muscle $\mathrm{Ca}^{2+}$ release channel (ryanodine receptor). Biophys J, 1995, 69: 106-119

107 Buratti R, Prestipino G, Menegazzi P, et al. Calcium dependent activation of skeletal muscle $\mathrm{Ca}^{2+}$ release channel (ryanodine receptor) by calmodulin. Biochem Biophys Res Commun, 1995, 213: 10821090

108 Board P G, Coggan M, Watson S, et al. CLIC-2 modulates cardiac ryanodine receptor $\mathrm{Ca}^{2+}$ release channels. Int J Biochem Cell Biol, 2004, 36: 1599-1612

109 Littler D R, Harrop S J, Goodchild S C, et al. The enigma of the CLIC proteins: Ion channels, redox proteins, enzymes, scaffolding proteins? FEBS Lett, 2010, 584: 2093-2101

110 Meng X, Wang G, Viero C, et al. CLIC2-RyR1 interaction and structural characterization by cryo-electron microscopy. J Mol Biol, 2009, 387: 320-334

111 Tripathy A, Resch W, Xu L, et al. Imperatoxin A induces subconductance states in $\mathrm{Ca}^{2+}$ release channels (ryanodine receptors) of cardiac and skeletal muscle. J Gen Physiol, 1998, 111: 679-690

112 Gurrola G B, Arevalo C, Sreekumar R, et al. Activation of ryanodine receptors by imperatoxin $\mathrm{A}$ and a peptide segment of the II-III loop of the dihydropyridine receptor. J Biol Chem, 1999, 274 : 7879-7886

113 Samso M, Trujillo R, Gurrola G B, et al. Three-dimensional location of the imperatoxin A binding site on the ryanodine receptor. J Cell Biol, 1999, 146: 493-499

114 Zhou Q, Wang Q L, Meng X, et al. Structural and functional characterization of ryanodine receptor-natrin toxin interaction. Biophys J, 2008, 95: 4289-4299

115 Maximciuc A A, Putkey J A, Shamoo Y, et al. Complex of calmodulin with a ryanodine receptor target reveals a novel, flexible binding mode. Structure, 2006, 14: 1547-1556 
116 Wright N T, Prosser B L, Varney K M, et al. S100A1 and calmodulin compete for the same binding site on ryanodine receptor. J Biol Chem, 2008, 283: 26676-26683

117 Balshaw D M, Xu L, Yamaguchi N, et al. Calmodulin binding and inhibition of cardiac muscle calcium release channel (ryanodine receptor). J Biol Chem, 2001, 276: 20144-20153

118 Yamaguchi N, Xu L, Pasek D A, et al. Molecular basis of calmodulin binding to cardiac muscle $\mathrm{Ca}^{2+}$ release channel (ryanodine receptor). J Biol Chem, 2003, 278: 23480-23486

119 Xiong L W, Newman R A, Rodney G G, et al. Lobe-dependent regulation of ryanodine receptor type 1 by calmodulin. J Biol Chem, 2002, 277: 40862-40870

120 Zhang H, Zhang J Z, Danila C I, et al. A noncontiguous, intersubunit binding site for calmodulin on the skeletal muscle $\mathrm{Ca}^{2+}$ release channel. J Biol Chem, 2003, 278: 8348-8355

121 Menegazzi P, Larini F, Treves S, et al. Identification and characterization of three calmodulin binding sites of the skeletal muscle ryanodine receptor. Biochemistry, 1994, 33: 9078-9084

122 Zorzato F, Fujii J, Otsu K, et al. Molecular cloning of cDNA encoding human and rabbit forms of the $\mathrm{Ca}^{2+}$ release channel (ryanodine receptor) of skeletal muscle sarcoplasmic reticulum. J Biol Chem, 1990, 265: 2244-2256

123 Chen S R, MacLennan D H. Identification of calmodulin-, $\mathrm{Ca}^{2+}-$, and ruthenium red-binding domains in the $\mathrm{Ca}^{2+}$ release channel (ryanodine receptor) of rabbit skeletal muscle sarcoplasmic reticulum. J Biol Chem, 1994, 269: 22698-22704

124 Most P, Remppis A, Pleger S T, et al. Transgenic overexpression of the $\mathrm{Ca}^{2+}$-binding protein $\mathrm{S} 100 \mathrm{~A} 1$ in the heart leads to increased in vivo myocardial contractile performance. J Biol Chem, 2003, 278: 33809-33817

125 Treves S, Scutari E, Robert M, et al. Interaction of S100A1 with the $\mathrm{Ca}^{2+}$ release channel (ryanodine receptor) of skeletal muscle. Biochemistry, 1997, 36: 11496-11503

126 Prosser B L, Wright N T, Hernandez-Ochoa E O, et al. S100A1 binds to the calmodulin-binding site of ryanodine receptor and modulates skeletal muscle excitation-contraction coupling. J Biol Chem, 2008, 283: 5046-5057

127 Du X J, Cole T J, Tenis N, et al. Impaired cardiac contractility response to hemodynamic stress in S100A1-deficient mice. Mol Cell Biol, 2002, 22: 2821-2829

128 McCarthy T V, Quane K A, Lynch P J. Ryanodine receptor mutations in malignant hyperthermia and central core disease. Hum Mutat, 2000, 15: 410-417

129 Amador F J, Liu S, Ishiyama N, et al. Crystal structure of type I ryanodine receptor amino-terminal beta-trefoil domain reveals a disease-associated mutation "hot spot" loop. Proc Natl Acad Sci USA, 2009, 106: 11040-11044

130 Lobo P A, Van Petegem F. Crystal structures of the N-terminal domains of cardiac and skeletal muscle ryanodine receptors: insights into disease mutations. Structure, 2009, 17: 1505-1514

131 Tung C C, Lobo P A, Kimlicka L, et al. The amino-terminal disease hotspot of ryanodine receptors forms a cytoplasmic vestibule. Nature, 2010, 468: 585-588

132 Lobo P A, Kimlicka L, Tung C C, et al. The deletion of exon 3 in the cardiac ryanodine receptor is rescued by beta strand switching. Structure, 2011, 19: 790-798

133 Wriggers W, Birmanns S. Using situs for flexible and rigid-body fitting of multiresolution single-molecule data. J Struct Biol, 2001, 133: 193-202

134 Wriggers W, Chacon P. Modeling tricks and fitting techniques for multiresolution structures. Structure, 2001, 9: 779-788

135 Baker M L, Serysheva II, Sencer S, et al. The skeletal muscle $\mathrm{Ca}^{2+}$ release channel has an oxidoreductase-like domain. Proc Natl Acad Sci USA, 2002, 99: 12155-12160

136 Wolfram F, Morris E, Taylor C W. Three-dimensional structure of recombinant type 1 inositol 1,4,5-trisphosphate receptor. Biochem J, 2010, 428: 483-489

137 Sato C, Hamada K, Ogura T, et al. Inositol 1,4,5-trisphosphate receptor contains multiple cavities and L-shaped ligand-binding domains. J Mol Biol, 2004, 336: 155-164

138 Serysheva II, Bare D J, Ludtke S J, et al. Structure of the type 1 inositol 1,4,5-trisphosphate receptor revealed by electron cryomicroscopy. J Biol Chem, 2003, 278: 21319-21322

139 da Fonseca P C, Morris S A, Nerou E P, et al. Domain organization of the type 1 inositol 1,4,5-trisphosphate receptor as revealed by single-particle analysis. Proc Natl Acad Sci USA, 2003, 100: 3936-3941

140 Hamada K, Terauchi A, Mikoshiba K. Three-dimensional rearrangements within inositol 1,4,5-trisphosphate receptor by calcium. J Biol Chem, 2003, 278: 52881-52889

141 Jiang Q X, Thrower E C, Chester D W, et al. Three-dimensional structure of the type 1 inositol 1,4,5-trisphosphate receptor at $24 \mathrm{~A}$ resolution. EMBO J, 2002, 21: 3575-3581

142 Bosanac I, Yamazaki H, Matsu-Ura T, et al. Crystal structure of the ligand binding suppressor domain of type 1 inositol 1,4,5trisphosphate receptor. Mol Cell, 2005, 17: 193-203

143 Chan J, Yamazaki H, Ishiyama N, et al. Structural studies of inositol 1,4,5-trisphosphate receptor: coupling ligand binding to channel gating. J Biol Chem, 2010, 285: 36092-36099

144 Bosanac I, Alattia J R, Mal T K, et al. Structure of the inositol 1,4,5-trisphosphate receptor binding core in complex with its ligand. Nature, 2002, 420: 696-700

145 Yuchi Z, Van Petegem F. Common allosteric mechanisms between ryanodine and inositol-1,4,5-trisphosphate receptors. Channels (Austin), 2011, 5: 120-123

146 Pettersen E F, Goddard T D, Huang C C, et al. UCSF Chimera--a visualization system for exploratory research and analysis. J Comput Chem, 2004, 25: 1605-1612

Open Access This article is distributed under the terms of the Creative Commons Attribution License which permits any use, distribution, and reproduction in any medium, provided the original author(s) and source are credited. 